\title{
Two-Dimensional High Performance Liquid Chromatography-Mass Spectrometry for Phosphatidylcholine Analysis in Egg Yolk
}

\author{
Justyna Walczak • Szymon Bocian • Bogusław Buszewski
}

Received: 29 April 2014 / Accepted: 11 July 2014 / Published online: 25 July 2014

(C) The Author(s) 2014. This article is published with open access at Springerlink.com

\begin{abstract}
Phospholipids are one of the classes of lipids, which are a major component of all cell membranes. Phosphatidylcholine (PC) is the main phospholipid present in egg yolk. The phospholipids extract from enriched egg yolk was separated using the two-dimensional high performance liquid chromatography in off-line system coupled with electrospray ionization tandem mass spectrometry (off-line HPLC-ESI$\mathrm{MS} / \mathrm{MS}$ ). In the first dimension, a preparative $\mathrm{C} 18$ column was used, and on the second dimension, a separation was performed on $\mathrm{N}, \mathrm{O}$-dialkylphosphoramidate bonded silica column. In the analyzed egg yolk, 15 different phosphatidylcholines was found. From among the identified fatty acid of PC, a large part constitutes docosahexaenoic acid ( $\omega-3,22: 6$, DHA) and linoleic acid ( $\omega-6,18: 2, \mathrm{LA})$.
\end{abstract}

Keywords Phosphatidylcholine - Omega fatty acids · Alkyl-phosphate bonded phase $\cdot$ LC-ESI-MS/MS

\section{Introduction}

Phospholipids (PLs) constitute complex and numerous group of organic compounds occurred in the all living organisms as the essential components of cellular membranes (lipid bilayer) and condensed energy as a spare material. A second function of phospholipids is to decrease triglycerides and cholesterol concentration in the blood and to prevent an accumulation of cholesterol in the vessel wall. Finally, PLs play crucial roles in signaling mechanisms for cell proliferation and cell death (Wright et al. 2004; Gładkowski et al. 2011). Moreover,

J. Walczak $\cdot$ S. Bocian $\cdot$ B. Buszewski $(\bowtie)$

Department of Environmental Chemistry and Bioanalytics, Faculty

of Chemistry, Nicolaus Copernicus University, Gagarin 7,

87-100 Torun, Poland

e-mail: bbusz@chem.umk.pl phospholipid metabolism plays an important role in numerous human diseases, cardiovascular, and cancer diseases, Alzheimer's and Parkinson's diseases and diabetes (Sato et al. 2010). Egg yolk included several classes of PLs: phosphatidylcholine (PC), phosphatidylethanolamine (PE), sphingomyelin (SM), lysophosphatidylcholine (LPC), lysophosphatidylethanolamine (LPE), and phosphatidylglycerol (PG) (Zhao et al. 2011). The most important phospholipid fraction of egg yolk are PC (73\%) and PE (15.5\%) (Anton 2007). Phosphatidylcholine is a component of lecithin. Lecithin has been isolated from egg yolk, due to the chemical composition, is more valuable than the lecithin of plant origin, since the composition contains up to $80 \%$ choline. It is known that choline is a main source of a precursor of the neurotransmitter-acetylcholine (Fiume 2001). Choline plays a role in liver function, prevention of cancer, and ensures the proper functioning of brain and development of memory (maintenance of learning memory performance) (Zeisel 1992; Zeisel 2000). Health-promoting properties of phospholipids are determined by the presence of omega-3 and omega- 6 fatty acids. Fatty acids above 20 carbon atoms is not found in the phospholipids isolated from plants materials. Therefore, phospholipids isolated from the egg yolk may be widely used in biomedical formulations. Omega-6 ( $\omega-6)$ and omega-3 ( $\omega-3)$ fatty acids, defined by the position of the double bond close to the methyl end of the molecule. Omega3 acids have the first double bond between the third methylene group and the rest of the molecule, while in omega- 6 acids, it is between the sixth methylene group and the rest of the molecule. The following acids belong to the omega-3 family: linolenic acid (ALA C18:3), eicosapentaenoic (EPA C20:5), docosapentaenoic (DPA C22:5), and docosahexaenoic (DHA C22:6). In omega-6 family, it may be found: linoleic acid (LA, C18:2) and arachidonic acid (AA, C20:4), respectively (Gładkowski et al., 2011; Le Grandois et al. 2009). The $\omega-3$ 
and $\omega-6$ fatty acids should be included in the diet or taken as supplements, because they are not synthesized by mammals (Gładkowski et al., 2011).

Phospholipids are analyzed by chromatographic techniques, such as the following: thin layer chromatography (TLC) (Romans and Palmer 1972), gas chromatography (GC) (Brondz 2002), and high performance liquid chromatography (Pacetti et al. 2005). High performance liquid chromatography (HPLC) is the most rapidly growing and very widely used modern techniques of separation. HPLC is characterized by high sensitivity and specificity, good resolution and high-speed process (Lima 2002). Phospholipids are determined by reversed-phase liquid chromatography (RP-LC) (Mazzella et al. 2004), normal-phase liquid chromatography (NP-LC) (Adlercreutz and Wehtje 2001), hydrophilic interaction liquid chromatography (HILIC) (Zhao et al. 2011), and multidimensional liquid chromatography (MDLC) (Lísa et al. 2011). There are various detectors available for use on conjunction with liquid chromatography. Commonly, natural lipids are monitored by low-wavelength UV detectors and refractive index detectors (RI) (Stith et al. 2000). However, UV response is highly dependent on the nature of fatty acid residues and varies with the degree of unsaturation within the same PL classes. Therefore, UV detection is not well adapted to the quantitative analysis of lipids with varying composition. From this reason, very popular are aerosol detectors, such as evaporative light-scattering detectors (ELDS), and charged aerosol detectors (CAD, Corona) (Ligor et al. 2013). In lipidomics mass spectrometry (MS) with different techniques of ionization, such as electronspray ionization (ESI), fast atom bombardment (FAB), and matrix-assisted laser desorption/ ionization (MALDI) are applied (Pulfer and Murphy 2003).

In this study, we propose an off-line LC $\times$ LC-ESI-MS/MS method using a triple quadrupole mass spectrometry for characterization of the fatty acids composition in phosphatidylcholines molecules in phospholipid extract from enriched egg yolk. In the study, a new stationary phase $N, O$ dialkylphosphoramidate (C18) was applied, which allow to separate phospholipids according to their polarity in reversedphase chromatography. The main goal of the work was the identification of phosphatidylcholines, which contains unsaturated fatty acids from omega- 3 and omega- 6 groups.

\section{Materials and Methods}

\section{Reagents}

Standard compounds of L- $\alpha$-phosphatidylcholine (PC), L- $\alpha$ phosphatidylethanolamine (PE), sphingomyelin (SM), L- $\alpha$ lysophosphatidylcholine (LPC), L- $\alpha-$ lysophosphatidylethanolamine (LPE), L- $\alpha-$ glycerophosphorylcholine (GPC), and L- $\alpha$ - lysophosphatidylglycerol (LPG) from egg yolk were purchased from Larodan Lipids (Malmö, Sweden). The methanol for HPLC was obtained from Sigma-Aldrich (Steinheim, Germany). Water was purified using Milli-Q RG apparatus (Millipore Intertech, Bedford, MA, USA) in our laboratory. Pure phospholipids fraction from egg yolk (Gładkowski et al. 2011; Gładkowski et al. 2012) was obtained from Wroclaw University of Environmental and Life Sciences.

Instrumentation

\section{First Dimension}

The apparatus was a Shimadzu Prominence HPLC system (Tokyo, Japan). Absorbance was measured at $206 \mathrm{~nm}$ using a diode array detector (SPD-M20A). The instrument includes a binary solvent delivery system (LC-20 AD), an autosampler (SIL-20A), and a column thermostat (CTO-10AS VP). In the first dimension, the Gemini NX C18 column $250 \times 10 \mathrm{~mm}$ with pore size $110 \AA$ (Phenomenex, Torrance, CA, USA) was used. The flow rate of the mobile phase was $1 \mathrm{~mL} / \mathrm{min}$, an injection volume was $10 \mu \mathrm{L}$, and separation temperature was $35{ }^{\circ} \mathrm{C}$. Pure methanol was used as a mobile phase. LabSolution software was used for data analysis. Fraction of phospholipid classes were collected manually. Each fraction was evaporated using Labconco CentriVap DNA concentrator (Kansas City, USA). Further, each fraction was dissolved in pure methanol to obtain concentration in the range of 90 $300 \mu \mathrm{g} / \mathrm{mL}$.

\section{Second Dimension}

Agilent model HPLC 1100 (Agilent, Waldbronn, Germany) was used as the chromatographic system. It consisted of a quaternary pump, a degasser, an automatic sample injection, and a variable wavelength $\mathrm{UV}-\mathrm{Vis}$ detector. Chromatographic system was coupled with the Agilent 6410 Triple Quad mass spectrometer (Agilent, Waldbronn, Germany) equipped with electrospray ionization (ESI) interface and operated with Mass Hunter software.

In the second dimension, collected fractions of phospholipids were analyzed using homemade $N, O$ dialkylphosphoramidate (C18) chemically bonded stationary phase (Bocian et al. 2012; Bocian et al. 2013). The short characteristic of the stationary phase is listed in Table 1 . The flow rate was $0.45 \mathrm{~mL} / \mathrm{min}$, the injection volume was $5 \mu \mathrm{L}$, and the separation temperature was $35{ }^{\circ} \mathrm{C}$. Analyses were done using isocratic mobile phase: $90 \%$ methanol and $10 \%$ water. Phosphatidylcholine (PC) was identified using ESI-MS in the mass range $m / z 30-1,000$ with the following setting of tuning parameters: pressure of the nebulizing gas $35 \mathrm{psi}$, the drying gas flow rate $8 \mathrm{~L} / \mathrm{min}$, and temperature of the drying gas $365^{\circ} \mathrm{C}$. For PC characterization in the positive mode, the 
Table 1 Physicochemical properties of N,O-dialkylphosphoramidate (C18) (Bocian et al. 2012)

\begin{tabular}{|c|c|}
\hline Structure & \\
\hline Functional group & Alkyl C18/phosphate/amine \\
\hline Dimension $[\mathrm{mm}]$ & $125 \times 4.6$ \\
\hline Silica particle size $[\mu]$ & 5 \\
\hline Pore diameter of bare silica $[\AA]$ & 100 \\
\hline Carbon content $[\%]$ & 9.33 \\
\hline Nitrogen content $[\%]$ & 1.15 \\
\hline Hydrogen content $[\%]$ & 2.20 \\
\hline Coverage density of alkyl ligands $\left[\mu \mathrm{mol} / \mathrm{m}^{2}\right]$ & 1.04 \\
\hline Coverage density of amine ligands $\left[\mu \mathrm{mol} / \mathrm{m}^{2}\right]$ & 2.80 \\
\hline
\end{tabular}

collision energy was set to $15 \mathrm{eV}$. PC species were manually identified with the parent mass information and their characteristic fragment ions in the CID spectrum using lists of PL species masses, published in the literature. Precursor scanning at $m / z 184$ was used for PC. Additionally, the position of given phospholipids on the chromatogram was confirmed by the comparison with phospholipids standard samples.

\section{Results and Discussion}

\section{Reserved-Phase Fractionation of Lipids}

Phospholipids' extract from egg yolk is a mixture of many compounds with similar chromatographic properties. Thus, it is impossible to separate particular compounds in traditional liquid chromatography. In this work, the off-line system of two dimensional liquid chromatography was applied. In first column, the preliminary separation of analyzed mixture at individual fraction was performed. The fractions were collected to eppendorf tubes and then the solvent was evaporated and again it was injected into second column (Herrero et al. 2009). RP-HPLC method has been applied for the analysis of lipids in the first dimension using C18 column and methanol as a mobile phase. The chromatogram obtained from the preparative column is shown in Fig. 1a. The first dimension did not provide satisfactory results. The main reason may be the sample complexity. The egg yolk is composed of variety phospholipids, which additionally differ in composition of fatty acids. Traditional separation using LC method usually leads to separation of individual lipid classes which differ in composition of acyl residues in the molecules (Mazzella et al. 2004). Separation mechanism of lipids on hydrophobic stationary phase has a partitioning nature which base on hydrophobic interactions. More hydrophobic lipids are strongly retained on the octadecyl stationary phase and elutes later from the column as compare to the more polar lipids. Elution order depends not only on the carbon chain length but also on the degree of unsaturation of acyl residues (Perona and RuizGutierrez 2003). Elution order of lipids in reversed phase are presented using the coefficient equivalent chain lengths (ECL) (Chen et al. 1999) and equivalent carbon number (ECN) for fatty acid and other lipids respectively (Perona and RuizGutierrez 2003). The equivalent chain length of fatty acid can be described by the following equation:

$\mathrm{ECL}=N-2 n$

where $N$ - carbon atom number and $n$ - the sum of double bonds in fatty acid molecule.

Each double bond reduces retention time, which is equal to shorten carbon length of saturated acid by two methylene units. Fatty acids that have similar ECL values are difficult to separate and have been referred to as "critical pairs" (for example, fatty acids $14: 0$ and $16: 1$ characterized by close retention time). The equivalent carbon number $(\mathrm{ECN})$ is defined as follows:

$\mathrm{ECN}=\mathrm{CN}-2 \mathrm{n}$

where $\mathrm{CN}$ is the sum of total carbon atom number of fatty acyl groups and $n$ is the sum of double bonds.

For example, 16:0/18:1-PC gives $\mathrm{ECN}=32$. The ECN and ECL coefficients of lipids to be resolved provide a valuable information for planning LC separation.

RP-HPLC Analysis of Phospholipids in Second Dimension

Thirteen fractions of PLs obtained from the first dimension have been analyzed by RP-HPLC coupled with mass spectrometry. 
Fig. 1 Chromatogram of egg yolk phospholipids separation: (a) first dimension; $\mathrm{C} 18$ column, mobile phase: $100 \% \mathrm{MeOH}$, flow $1 \mathrm{~mL} / \mathrm{min}$, (b) second dimension; $\mathrm{N}, \mathrm{O}$ dialkylphosphoramidate (C18), mobile phase: $90 \% \mathrm{MeOH} / 10 \%$ $\mathrm{H}_{2} \mathrm{O}$, flow $0.45 \mathrm{~mL} / \mathrm{min}$. Number of collected fractions in the chromatogram a corresponds to number of chromatograms in the b
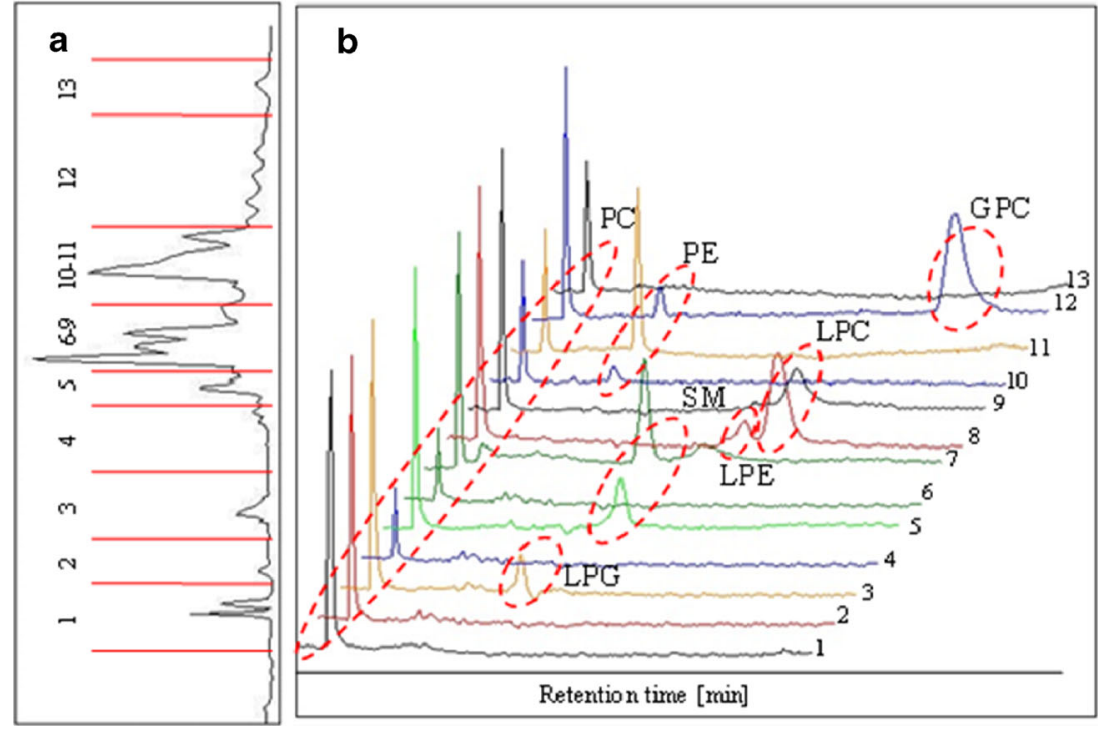

$\mathrm{N}, \mathrm{O}$-dialkylphosphoramidate $(\mathrm{C} 18)$ stationary phase was used. The analyzed phospholipids consisted of phosphatidylcholine PC (1), phosphatidylethanolamine PE (2), lysophosphatidylglycerol LPG (3), sphingomyelin SM (4), lysophosphatidylethanolamine LPE (5), lysophosphatidylcholine LPC (6), and glycerophosphorylcholine GPC (7). The identified classes of phospholipids are shown in Fig. 1b. Each fraction contains phosphatidylcholine, because egg's phospholipids are mainly composed of phosphatidylcholine (73\%) and phosphatidylethanolamine (15.5\%) (Anton 2007). PC species are separated according to the acyl chain lengths and the number of double bonds (Eq. 2). The retention time of phospholipids increases proportionally to ECN coefficient. PC species which contain fatty acyls with five and six double bonds are retained more strongly, and they elute in higher ECN groups. Analysis of phospholipids in egg yolk with $\mathrm{N}, \mathrm{O}$-dialkylphosphoramidate (C18) stationary phase allow to separate phospholipids with respect to polar part of the molecule. It suggest that separation mechanism is based on polar interaction of given molecules with the stationary phase surface, despite the fact that the separation is carried out in reversed-phase condition. It is possible due to polar-embedded groups in the structure of bonded ligands (Bocian et al. 2013). In the first dimension, separation is based on hydrophobicity of fatty acids/or hydrophobic interactions with stationary phase (differences in chain lengths and number of double bonds of acyl residues). In the second dimension (with the retention time on Fig. 1b), separation based on the differences in the polarity of the phospholipid "headgroups" takes place.

The $N, O$-dialkylphosphoramidate $(\mathrm{C} 18)$ stationary phase, depending on the composition of mobile phase, can be used in reversed phase (RP) and hydrophilic interaction liquid chromatography (HILIC). The unique nature of this material results from specific properties of adsorbent. The silica surface, amine, and phosphate groups are polar, and organic octadecyl chains are hydrophobic. Application of $N, O$ dialkylphosphoramidate $(\mathrm{C} 18)$ stationary phase for the phospholipids analysis is a good solution, because of the structure similarities between bonded ligands and phospholipids (a polar "head" and nonpolar tails).

Identification of Individual Molecular Species of Phosphatidylcholine

Phospholipids belong to zwitterionic molecules. They ionize in both positive and negative mode. The deprotonated molecules in negative-ion ESI mass spectra are formed by phosphatidylethanolamine, lysophosphatidylethanolamine, phosphatidylglycerol, phosphatidylinositol, and phosphatidylserine, respectively,
Fig. 2 The fragmentation pathway for the formation of $\mathrm{m} / \mathrm{z}$ 184 ion, proposed by Murphy et al. (Murphy and Axelsen 2011)

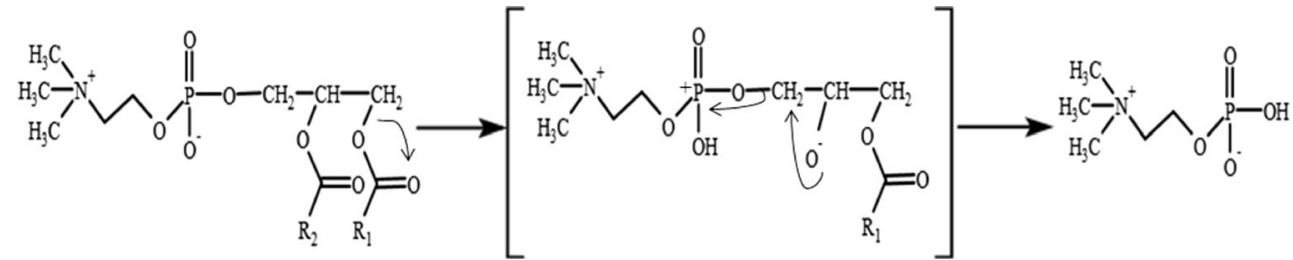



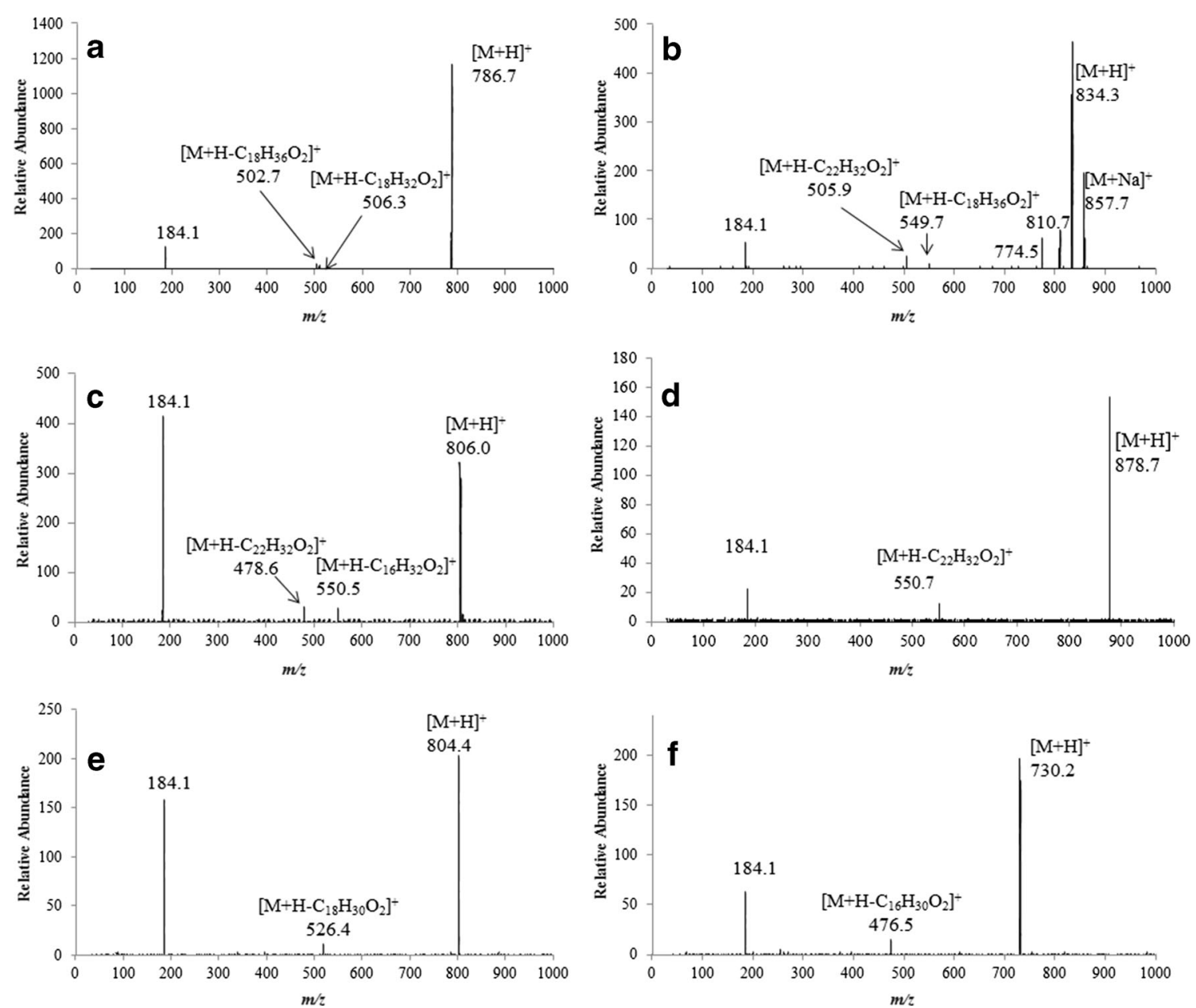

Fig. 3 Product ion scan in positive mode ESI-MS/MS of phosphatidylcholine: (a) $[\mathrm{M}+\mathrm{H}]^{+}$786.7, (b) $[\mathrm{M}+\mathrm{H}]^{+} 843.3,(\mathbf{c})[\mathrm{M}+\mathrm{H}]^{+} 806.6,(\mathbf{d})[\mathrm{M}+\mathrm{H}]^{+}$ 878.7, (e) $[\mathrm{M}+\mathrm{H}]^{+}$804.4, (f) $[\mathrm{M}+\mathrm{H}]^{+} 730.2$

giving $[\mathrm{M}-\mathrm{H}]^{-}$ions. Phospholipids with choline head (phosphatidylcholine, lysophosphatidylcholine, and sphingomyelin) in the positive-ion mode generate protonated $[\mathrm{M}+\mathrm{H}]^{+}$ions. However, for the phosphatidylethanolamine, it could be observed
Table 2 Characterization of phosphatidylcholine molecular species in lipid extract from egg yolk. Fatty acids from $\omega-3$ and $\omega-6$ are shown in italic in the table

\begin{tabular}{llllll}
\hline Molecular species & Theoretical $m / z$ & Measurement $m / z$ & {$\left[\mathrm{M}+\mathrm{H}-\mathrm{R}_{1}\right]^{+}$} & {$\left[\mathrm{M}+\mathrm{H}-\mathrm{R}_{2}\right]^{+}$} & $\mathrm{ECN}$ \\
\hline C16:1-C16:1 & 730.6 & 730.2 & 476.5 & 476.5 & 28 \\
C16:0-C16:0 & 734.6 & 734.4 & 478.5 & 478.5 & 32 \\
C16:0-C18:2 & 758.6 & 758.5 & 502.5 & 478.0 & 30 \\
C16:0-C18:1 & 760.7 & 760.7 & 504.2 & 478.2 & 32 \\
C16:0-C18:0 & 762.6 & 762.3 & 506.3 & 478.8 & 34 \\
C18:1-C18:2 & 784.6 & 784.4 & 504.0 & 502.4 & 30 \\
C18:0-C18:2 & 786.7 & 786.7 & 502.7 & 506.3 & 32 \\
C18:0-C18:0 & 790.7 & 790.7 & 506.2 & 506.2 & 36 \\
C16:3-C22:6 & 802.6 & 802.4 & 474.4 & 552.4 & 20 \\
C18:3-C20:5 & 804.6 & 804.4 & 501.9 & 526.4 & 22 \\
C16:0-C22:6 & 806.6 & 806.0 & 478.6 & 550.5 & 26 \\
C18:0-C22:6 & 834.7 & 834.3 & 505.9 & 549.7 & 28 \\
C20:3-C22:6 & 856.7 & 856.4 & 528.4 & 550.4 & 24 \\
$C 22: 6-C 22: 6$ & 878.7 & 878.2 & 550.7 & 550.7 & 20 \\
C22:5-C22:6 & 880.7 & 880.4 & 550.7 & 552.7 & 22 \\
\hline
\end{tabular}


both protonated and deprotonated ions (Murphy and Axelsen 2011). In order to characterize individual molecular species of PC, phospholipids extracts from enriched hen's egg yolk were analyzed by ESI-Q ${ }^{\mathrm{n}}$ MS in positive mode. Furthermore, the theoretical masses from literature were used in order to identify fatty acid attached to the PC molecules. In a single stage MS mode, it was observed only $[\mathrm{M}+\mathrm{H}]^{+}$ions and alkali metal adducts ions such as $[\mathrm{M}+\mathrm{Na}]^{+}$(Murphy and Axelsen 2011).

Fragmentation mass spectra $\mathrm{MS}^{2}$, give an intense signal derived from protonated phosphocholine head $\mathrm{m} / \mathrm{z} 184.1$ $\left[\mathrm{C}_{5} \mathrm{H}_{14} \mathrm{NPO}_{4}+\mathrm{H}\right]^{+}$. The fragmentation pathway for the formation of $m / z 184$ ion, was proposed by Murphy et al. (Murphy and Axelsen 2011) and is shown in Fig. 2. The phosphocholine ion is formed by the transfer of a proton from the $s n-2$ group to the phosphate group. Figure 3 presents the product ion mass spectra of some identified PC molecular species. Figure 3 a shows the mass spectra of the ion molecular $[\mathrm{M}+\mathrm{H}]^{+}$at $\mathrm{m} / \mathrm{z}$ 786.7. In the spectrum, signals at $\mathrm{m} / \mathrm{z} 506.3$ and $\mathrm{m} / \mathrm{z} 502.7$ were also observed. These $\mathrm{m} / \mathrm{z}$ values correspond to polar headgroup and two acyl groups, C18:2 and C18:0, respectively. For PC 18:0/22:6 (Fig. 3b), the $\mathrm{MS}^{2}$ fragmentation of the selected ion $[\mathrm{M}+\mathrm{H}]^{+}$at $m / z 834.3$ show the loss of two ions, at $\mathrm{m} / \mathrm{z} 549.7$ (minor) and $\mathrm{m} / \mathrm{z} 505.9$ (major), which corresponds to stearic and docosahexaenoic acid, respectively. Additionally, in Fig. 3b, sodium adduct $[\mathrm{M}+\mathrm{Na}]^{+}$can be observed at $\mathrm{m} / \mathrm{z}$ 857.7. Alkali metal may appear as a result of contamination of the entire system. Except the major ion of $\mathrm{m} / \mathrm{z}$ 184, the product ion spectrum of PC 16:0/22:6 (Fig. 3c), contains ions at $m / z 478.6\left[\mathrm{M}+\mathrm{H}-\mathrm{C}_{22} \mathrm{H}_{32} \mathrm{O}_{2}\right]^{+}$and $m / z 550.5$ $\left[\mathrm{M}+\mathrm{H}-\mathrm{C}_{16} \mathrm{H}_{32} \mathrm{O}_{2}\right]^{+}$, arising from elimination of the fatty acid from the molecule. A similar ion at $\mathrm{m} / \mathrm{z} 551.7[\mathrm{M}+\mathrm{H}-$ $\left.\mathrm{C}_{22} \mathrm{H}_{32} \mathrm{O}_{2}\right]^{+}$was observed for PC 22:6/22:6 (Fig. 3d), indicating that the loss of free fatty acid $(\mathrm{C} 22: 6)$ involves the $\mathrm{H}^{+}$ion that protonated the molecule to a $[\mathrm{M}+\mathrm{H}]^{+}$. In the case of $\mathrm{PC}$ 18:3/20:5 (Fig. 3e), the fragment at $m / z 526.4$ was described for ion $\left[\mathrm{M}+\mathrm{H}-\mathrm{C}_{18} \mathrm{H}_{30} \mathrm{O}_{2}\right]^{+}$, which constitute the $\alpha$-linolenic acid. Figure $3 f$ shows the mass spectra of PC 16:1/16:1. The major signal on this spectrum is the molecular ion $[\mathrm{M}+\mathrm{H}]^{+}$, detected as $m / z 730.2$ (two molecules of palmitoleic acid).

During the study, 15 phosphatidylcholine molecular species from enriched hen's egg yolk were identified (Table 2). Table 2 also presents the equivalent carbon number (ECN) for each PC molecules. All these identified molecular species contain fatty acids with chain lengths varying from 16 to 22 carbon atoms and the double bond number raining from 1 to 6 . From among the identified fatty acid of PC, a large part constitute polyunsaturated fatty acid (PUFA), which include fatty acids of omega- 3 and omega- 6 . The role of PUFAs is very important, because they are not synthesized in human organism and have to be delivered with food (Gładkowski et al. 2012). Docosahexaenoic acid (22:6, DHA) and eicosapentaenoic acid (20:5, EPA) belongs to $\omega-3$ family of fatty acid and are necessary for brain and eyesight development (Guesnet and Alessandri 2011; Uauy and Dangour 2006). Linolenic acid (18:3, ALA) protects skin from atopic dermatitis (Van Goll, et al. 2004). To $\omega-6$ family of fatty acids belongs linoleic acid (18:2, LA) and docosapentaenoic acid (22:5). Among 15 identified PC species, 7 contains $\omega-3$ fatty acids. Two of them contain also $\omega-6$ fatty acids. Another two PCs contain one $\omega-6$ fatty acid in the molecule.

\section{Conclusions}

In this study, off-line LC $\times$ LC-ESI-MS/MS method has been used to identify 15 molecular species of egg yolk phosphatidylcholine. The multidimensional liquid chromatography is a useful technique in analysis and separation of complex mixtures such as phospholipids. In the second dimension homemade $\mathrm{N}, \mathrm{O}$-dialkylphosphoramidate $(\mathrm{C} 18)$ column was used. This stationary phase exhibits good selectivity in analysis of phospholipids from egg yolk, according to polar head in RP conditions. The results show the presence of acids from the group of $\omega-3$ and $\omega-6$ in PC molecules. Among 15 identified PCs, 7 species contains $\omega-3$ fatty acids. In two cases the combination of $\omega-3$ and $\omega-6$ fatty acids was observed. Another two PCs contain one $\omega-6$ fatty acid in the molecule.

Acknowledgments This work was supported by Faculty of Chemistry NCU 2013 grant for young scientists no. 1519-Ch. Synthesis of $\mathrm{N}, \mathrm{O}-$ dialkylphosphoramidate (C18) stationary phase was supported by Ministry of Science and Higher Education, Grant No. NCN 2013/09/D/ST4/ 03807 for period 2014-2016.

Conflict of Interest Justyna Walczak, Szymon Bocian, and Bogusław Buszewski declare that they have no conflict of interest. This article does not contain any studies with human or animal subjects.

Open AccessThis article is distributed under the terms of the Creative Commons Attribution License which permits any use, distribution, and reproduction in any medium, provided the original author(s) and the source are credited.

\section{References}

Adlercreutz D, Wehtje E (2001) A simple HPLC method for the simultaneous analysis of phosphatidylcholine and its partial hydrolysis products 1- and 2-acyl lysophosphatidylcholine. J Am Oil Chem Soc 78(10):1007-1011

Anton M (2007) Composition and structure of hen egg yolk. In: Huopalathi R, Lopez-Fandino R, Anton R, Schade R (eds) Bioactive egg compounds. Springer Verlag Berlin, Heidelberg, pp $1-6$

Bocian S, Nowaczyk A, Buszewski B (2012) New alkyl-phosphate bonded stationary phases for liquid chromatographic separation of biologically active compounds. Anal Bioanal Chem 404:731-730 
Bocian S, Paca M, Buszewski B (2013) Characterization of new N, Odialkyl phosphoramidate-bonded stationary phases for reversedphase HPLC - retention and selectivity. Analyst 128:5221-5229

Brondz I (2002) Development of fatty acid analysis by high-performance liquid chromatography, gas chromatography, and related techniques. Anal Chim Acta 465(1-2):1-37

Chen SH, Chen KC, Lien HM (1999) Determination of fatty acids in vegetable oil by reversed phase liquid chromatography with fluorescence detection. J Chromatogr A 849(2):357-369

Fiume Z (2001) Final report on the safety assessment of lecithin and hydrogenated lecithin. Int J Toxicol 1:21-45

Gładkowski W, Kiełbowicz G, Chojnacka A, Gil M, Trziszka T, Dobrzański Z, Wawrzeńczyk C (2011) Fatty acid composition of egg yolk phospholipids fractions following feed supplementation of Lohmann Brown hens with humic-fat preparations. Food Chem 126:1013-1018

Gładkowski W, Chojnacka A, Kiełbowicz G, Trziszka T, Wawrzeńczyk C (2012) Isolation of pure phospholipid fraction from egg yolk. J Am Oil Chem Soc 89:179-182

Guesnet P, Alessandri JM (2011) Docosahexaenoic acid (DHA) and the developing central nervous system (CNS)-Implications for dietary recommendations. Biochimie 93(1):7-12

Herrero M, Ibáńez E, Cifuentes A, Bernal J (2009) Multidimensional chromatography in food analysis. J Chromatogr A 1216(43):7110-7129

Le Grandois J, Marchioni E, Zhao M, Giuffrida F, Ennahar SD, Bindler F (2009) Investigation of natural phosphatidylcholine sources: separation and identification by liquid chromatography-electrospray ionization-tandem mass spectrometry (LC-ESI-MS ${ }^{2}$ ) of molecular species. J Agric Food Chem 57:6014-6020

Ligor M, Studzińska S, Horna A, Buszewski B (2013) Corona-charged aerosol detection: an analytical approach. Crit Rev Anal Chem 43: 64-78

Lima A (2002) High-performance liquid chromatography of fatty acids in biological samples. Anal Chim Acta 465:81-91

Lísa M, Cífková E, Holcapek M (2011) Lipidomic profiling of biological tissues using offline two-dimensional high performance liquid chromatography-mass spectrometry. J Chromatogr A 1218:5146-5156

Mazzella N, Molinet J, Syakti AD, Dodi A, Doumenq P, Artaud J, Bertrand JC (2004) Bacterial phospholipid molecular species analysis by ion-pair reversed-phase HPLC/ESI/MS. J Lipid Res 45(7): $1355-1363$
Murphy RC, Axelsen PH (2011) Mass spectrometric analysis of longchain lipids. Mass Spectrom Rev 30:579-599

Pacetti D, Boselli E, Hulan HW, Frega NG (2005) High performance liquid chromatography-tandem mass spectrometry of phospholipid molecular species in eggs from hens fed diets enriched in seal blubber oil. J Chromatogr A 1097:66-73

Perona JS, Ruiz-Gutierrez V (2003) Simultaneous determination of molecular species of monoacylglycerols, diacylglycerols and triacylglycerols in human very low density lipoproteins by reversed phase liquid chromatography. J Chromatogr B 785(1):89-99

Pulfer M, Murphy RC (2003) Electrospray mass spectrometry of phospholipids. Mass Spectrom Rev 22(5):332-364

Romans JR, Palmer IS (1972) Photoreflectometric method for the quantitative analysis of neutral lipids after thin-layer chromatography. Anal Biochem 49(2):580-584

Sato Y, Nakamura T, Aoshima K, Oda Y (2010) Quantitative and wide ranging profiling of phospholipids in human plasma by twodimensional liquid chromatography/mass spectrometry. Anal Chem 82:9858-9864

Stith BJ, Hall J, Ayres P, Waggoner L, Moore JD, Shaw WA (2000) Quantification of major classes of Xenopus phospholipids by high performance liquid chromatography with evaporative light scattering detection. J Lipid Res 41(9):1448-1454

Uauy R, Dangour AD (2006) Nutrition on brain development and aging role of essential fatty acids. Nutr Rev 64(5):24-33

Van Goll CJ, Zeegers MP, Thijs C (2004) Epidemiology and health services research. Oral essential fatty acid supplementation in atopic dermatitis a meta-analysis of placebo controlled trials. Br J Dermatol 150:728-740

Wright MM, Howe AG, Zaremberg V (2004) Cell membranes and apoptosis: role of cardiolipin, phosphatidylcholine, and anticancer lipid analogues. Biochem Cell Biol 82(1):18-26

Zeisel SH (1992) Choline: an important nutrient in brain development, liver function and carcinogenesis. J Am Coll Nutr 11:473-481

Zeisel SH (2000) Choline: needed for normal development of memory. J Am Col Nutr 19:528-553

Zhao Y-Y, Xiong Y, Curtis JM (2011) Measurement of phospholipids by hydrophilic interaction liquid chromatography coupled to tandem mass spectrometry: the determination of choline containing compounds in foods. J Chromatogr A 1218:5470-5479 